February 2022

\title{
Revisit hospitality and tourism research rankings in six journals: Second decade of the new millennium
}

\author{
Yuehan Cao \\ lowa State University, ycao@iastate.edu \\ Jiyeon Jeon \\ lowa State University, jjeon@iastate.edu \\ Liang R. Tang \\ lowa State University, rebeccat@iastate.edu \\ See next page for additional authors \\ Follow this and additional works at: https://digitalcommons.usf.edu/jght \\ Part of the Hospitality Administration and Management Commons, and the Tourism and Travel

\section{Commons} \\ This Refereed Article is brought to you for free and open access by the M3 Center at the \\ University of South Florida Sarasota-Manatee at Digital Commons @ University of South Florida. \\ It has been accepted for inclusion in Journal of Global Hospitality and Tourism by an authorized \\ editor of Digital Commons @ University of South Florida. For more information, please contact \\ digitalcommons@usf.edu.
}

\section{Recommended Citation}

Cao, Y., Jeon, J., Tang, L. R., \& Wang, X. (2022). Revisit hospitality and tourism research rankings in six journals: Second decade of the new millennium. Journal of Global Hospitality and Tourism, 1(1), 1-15. https://www.doi.org/10.5038/2771-5957.1.1.1000

Corresponding Author

Liang(Rebecca) Tang, 12 Mackay Hall, 2302 Osborn Dr., Dept. of Apparel, Events, \& Hospitality Management, lowa

State University, Ames, IA, U.S. 50011-1078

Revisions

Submission date: Jul. 12, 2021; 1st Revision: Nov. 3, 2021; Acceptance: Dec. 6, 2021 
Revisit hospitality and tourism research rankings in six journals: Second decade of the new millennium

\section{Authors}

Yuehan Cao, Jiyeon Jeon, Liang R. Tang, and Xi Wang 


\title{
Revisit Hospitality and Tourism Research Rankings in Six Journals: Second Decade of the New Millennium
}

\author{
Yuehan $\mathrm{Cao}^{1}$, Jiyeon Jeon ${ }^{2}$, Liang (Rebecca) Tang ${ }^{3}$, and Xi Wang ${ }^{4}$
}

College of Human Sciences

Iowa State University, USA

1ycao@iastate.edu

2jjeon@iastate.edu

${ }^{3}$ rebeccat@iastate.edu

\author{
Division of Culture and Creativity \\ BNU-HKBU United International College, China \\ 4xiwang@uic.edu.cn
}

\begin{abstract}
The purpose of this research was to replicate an earlier study that analyzed hospitality and tourism research contributions in the first decade of the new millennium (Park et al., 2011) using a time frame of 2010-2019. The present study reviewed six top-tier hospitality and tourism journals, including the International Journal of Hospitality Management, Journal of Hospitality \& Tourism Research, Cornell Hospitality Quarterly, Tourism Management, Journal of Travel Research, and Annals of Tourism Research. The papers were classified by research themes in the hospitality and tourism categories, respectively. The results offered the rankings by publication productivity pertaining to individual authors, universities, and countries/regions across the world. The findings give insights into future directions and identify research needs for academicians and industry practitioners in the hospitality and tourism field.
\end{abstract}

Keywords: rankings, authors, universities, countries/regions, journals

\section{Introduction}

In the new millennium, the hospitality and tourism field has witnessed tremendous revolutions corresponding to the changes in the global environment from the perspectives of technology, society, economy, culture, and others (Uysal et al., 2017). Consequently, the hospitality and tourism discipline has experienced exponential augmentation in journals, publication opportunities, and cooperation (Gursoy \& Sandstrom, 2016). In more recent years, the Web of Science even identified hospitality and tourism as an independent academic category, which further confirmed the progression of the discipline (Min et al., 2016). Considering the marvelous changes in the industry and academic maturity, an opportune moment occurred to review the progression of research scholarship in the hospitality and tourism field.

As Kim et al. (2018, p.49) suggested, 'for a field to progress, it must be conscious of its historical patterns to obtain insights into possible future development and implications that contribute to the accumulation of knowledge.' Thus, it is essential to review the literature of a field periodically 
(Line \& Runyan, 2012). However, the majority of previous review studies in the hospitality and tourism field vary in scope and comprehensiveness, such as the adoption of distinct variables (e.g., research themes, journals), methods (e.g., qualitative vs. quantitative), and time period for the journal paper published. Thus, they mostly serve as independent pieces and cannot be integrated to demonstrate a thorough history. Thus, we argued it is crucial to examine the historical pattern of literature periodically with the consistent method.

Park et al. (2011)'s study is such an exemplary review study. This research continued in the same vein with multiple review studies in the 1990s and 2000s (i.e., Sheldon, 1991; Jogaratnam et al., 2005a; Jogaratnam et al., 2005b). Specifically, Park et al. (2011) classified the papers by research themes in hospitality and tourism respectively and further gave rankings by the productivity of authors, universities, and countries/regions using research contributions to six top-tier journals in the first decade of the new millennium. The disaggregation of the papers based on their respective themes could help scholars generate a more accurate and deep understanding of the relative importance (McKercher et al., 2006). Furthermore, rankings offer a practical tool for measuring scholarship quality (McKercher, 2012). Within a university, administrators entail immense knowledge of scholar rankings to better assess the performance and productivity of faculty members and hire faculty whose research agenda are consistent with the missions of the programs, and promote inter-disciplinary scholarship (Ip et al., 2011). The rankings by individual institutes are frequently considered in the accrediting process of the programs (Jogaratnam et al., 2005). The standing of an individual discipline in a country/region is a component of gauging its overall quality of the higher education institute system (Shen et al., 2018). Acknowledging the importance of rankings used in Park et al. (2011), we conducted a follow-up study with the same method by reviewing the papers published during the second decade of the new millennium. Together with Park et al. (2011), the present study gave a comprehensive overview of the historical patterns that have sustained the progression of research scholarship in the hospitality and tourism field in the new millennium.

The current study has three specific objectives: 1) to classify the papers in hospitality and tourism respectively by themes, 2) to offer rankings by most productive authors, universities, and countries/regions, and 3) compare the results between the period of 2010-2019 analyzed in the present study and the period of 2000-2009 shown in Park et al. (2011). The present study is expected to assist scholars and practitioners in better understanding the current state of hospitality and tourism research and predict directions for future studies.

\section{Previous Review Studies in Hospitality and Tourism}

Previous review studies were classified into two streams. The first approach was to investigate a specific research theme by reviewing multiple journals. For example, Chan and Hsu (2016) conducted topical reviews of 149 research papers related to the green and environmental issues published during 1993-2006. Singh et al. (2007) did a content analysis of the research papers in human resource management (HRM) from 1994 to 2003. Similarly, Tracey (2014) compared the studies in HRM published in discipline-based and hospitality-specific journals. Other themes which got special attention in the review papers of the hospitality and tourism field included diversity and diversity management (Manoharan \& Singal, 2017), marketing (e.g., Line \& Runyan, 2012), corporate social responsibility (Serra-Cantallops et al., 2018), operation management (Jones \& Lockwood, 1998), accounting and finance (Harris \& Brown, 1998), strategic 
management (Olsen \& Roper, 1998), and others. The second approach was to give a systematic review, analysis, and evaluation of all the research papers published in one or several journals. There are examples that reviewed papers in a specific journal. Specifically, Law et al. (2012) did a content analysis of the papers published in Cornell Hospitality Quarterly during 2008-2011. Cunill et al. (2019) offered a lifetime overview of the papers published in the International Journal of Hospitality Management to celebrate its 35th anniversary in 2019. Lu and Nepal (2009) reviewed the papers published in the Journal of Sustainable Tourism during 1993-2007. Law et al. (2012) conducted an assessment of research papers in Cornell Hospitality Quarterly during 20082011.

Most of the studies which review multiple journals were independent pieces. For example, Svensson et al. (2009) investigated the distributions of empirical and non-empirical papers published in six hospitality and tourism journals. Ryan (2005) gave the ratings of 22 journals in tourism, leisure, and sports research. Different from these independent review studies, Sheldon (1991), Jogaratnam et al. (2005a), Jogaratnam et al. (2005b), and Park et al. (2011) formed a chain to demonstrate the historical pattern of the hospitality and tourism field with similar methods. Specifically, Sheldon (1991) analyzed the authorship of research published in three tourism journals during the 1980s and reported the positions of the most productive authors and proportions of these authors across various geographic regions. Jogaratnam et al. (2005a) replicated Sheldon (1991)'s method and reviewed three tourism journals in the time frame of 1992-2001. Similarly, Jogaratnam et al. (2005b) analyzed 11 hospitality and tourism journals during 1992-2001 and ranked the top 20 universities and five continents which contributed the most. To take the idea further, Park et al. (2011) conducted a more detailed classification of research topics and ranked the top scholars, universities, and countries/regions during 2000-2009.

\section{Methods}

\section{Data Collection}

Consistent with Park et al. (2011), we collected all the research papers and research notes, including those in special issues published in the International Journal of Hospitality Management (IJHM), Journal of Hospitality and Tourism Research (JHTR), Cornell Hospitality Quarterly (CHQ), Tourism Management (TM), Journal of Travel Research (JTR), and Annals of Tourism Research (ATR) during 2010-2019. We created a database for analysis, which was composed of authors, their affiliated universities, and countries/regions of these universities, as well as the research themes that individual papers were categorized into.

\section{Data Analysis}

The present study adopted the fractional score mechanism. Specifically, one point was assigned to each paper. Then the one point was equally distributed among the authors, their affiliated universities, and the countries/regions these universities are located. For example, Çakmak et al. (2018) published a paper in ATR. At the time of the publication, Çakmak belonged to Breda University of Applied Sciences, Lie belonged to Wageningen University, and McCabe belonged to Nottingham University. University of Applied Sciences and Wageningen University are in the Netherlands, while Nottingham University is in the United Kingdom. Thus, each of the three authors got one-third of a point. Each of the three universities gained one-third of a point. The 
Netherlands got two-third of a point, while the United Kingdom obtained one-third of a point. The fractional score method has also been widely applied in different contexts to measure the rankings. For instance, Adler et al. (2002) used it to measure the ranking of marketing journals, Nusair et al. (2019) adopted it to assess the ranking of social media research, Severt et al. (2009) used it to measure hospitality and tourism programs, and Zhou and Leydesdorff (2011) adopted it to evaluate the research output among academic programs.

We classified each paper into either hospitality or tourism. Furthermore, the hospitality papers were classified into 11 themes: accounting and finance (ACF), education (EDU), green and environmental issue (ENV), food service management (FSM), human resource management (HRM), information technology and management information system (IT/MIS), legal issue (LAW), marketing (MKT), operating management (ORM), strategic management (STM), and others (OTH). The tourism papers were classified into 20 themes, including attraction management (ATT), crisis and safety management (CSM), destination marketing and management (DMKT), tourism development (DVP), economic impact and econometrics (ECO), education (EDU), geographical issue (GEO), general marketing (GMKT), image and branding (IMG), information technology (IT), meetings, incentives, conventions, and exhibitions including festival and fair (MICE), tourism planning (PLN), politics, policy, legal, and governmental issue (PPL), supply chain management (SCM), segmentation (SEG), special interests tourism such as heritage, farm, cultural, wine, or food tourism (SIT), service management (SMT), sustainable tourism and ecotourism (SUT), tourists' perception and behavior (TPB), and others (OTH). Two coders independently categorized each sample to either hospitality or tourism and then identified the research theme the best. It is possible that some papers covered multiple themes, and the coders identified the theme that the paper was best fit in. Inter rater reliability check was adopted to test the consistency of the two coders' results. Cronbach's alpha as an indicator of inter-coder reliability for the hospitality papers was .98 , while that for the tourism papers was .99 , which were both acceptable (Tinsley and Brown, 2000). For the discrepancies of the initial results between the two coders, another senior researcher in hospitality and tourism was involved in the discussions and decided the final results with them together.

We reported the rankings of 50 authors, 50 universities, and 20 countries/regions that contributed most with the fractional score mechanism for hospitality and tourism, respectively. And then, we combined hospitality and tourism papers together and accordingly ranked the top 100 authors, 100 universities, and 30 countries/regions that contributed most. Particularly, since the number of publications in an individual program/department/college of a university is highly related to the quantity of faculty members, weight scores were also offered to reduce size bias. The number of faculty members covered the academic staff but excluded adjunct, visiting, and emeritus professors, which was gained from the webpage of each program during April 16-30, 2020.

\section{Results}

\section{Descriptive Information}

The summary of the hospitality and tourism papers is demonstrated in Table 1. The total papers published in the six journals enhanced from 2,834 during the first decade to 5,358 during the second decade of the millennium, with the increasing rate of $89.1 \%$. The three hospitality journals published 2,000 papers during 2010-2019 comparing to 1,082 ones during 2000-2009, while the 
three tourism journals published 3,358 papers during 2010-2019 comparing to 1,752 ones during 2000-2009. Among the six journals, TM published the most papers, and JHTR published the least ones. IJHM published the most papers relevant to hospitality, while TM published the most papers relevant to tourism during 2010-2019. The results were consistent with those during 2000-2009. The number of total authors who appeared in these journal papers during 2010-2019 was 14,064, with a $136 \%$ increase from that during 2000-2009. However, the average number of authors for each paper was 2.74 during 2010-2019, similar with 2.10 during 2000-2009.

Table 1. Research Summary During 2010-2019

\begin{tabular}{lccccc}
\hline Journal & $\begin{array}{c}\text { Number of } \\
\text { Hospitality Papers }\end{array}$ & $\begin{array}{c}\text { Number of } \\
\text { Tourism Papers }\end{array}$ & $\begin{array}{c}\text { Total Number } \\
\text { of Papers }\end{array}$ & $\begin{array}{c}\text { Total Appeared Number } \\
\text { of Authors }\end{array}$ & $\begin{array}{c}\text { Average Number of Authors } \\
\text { Per Paper }\end{array}$ \\
\hline JHTR & 163 & 168 & 331 & 837 & 2.53 \\
IJHM & 1,235 & 133 & 1,368 & 3,685 & 2.69 \\
CHQ & 314 & 26 & 340 & 866 & 2.43 \\
Subtotal & 1,712 & 327 & 2,039 & 5,388 & 2.55 \\
TM & 238 & 1,514 & 1,752 & 4,803 & 2.74 \\
ATR & 17 & 921 & 938 & 2,163 & 2.31 \\
JTR & 33 & 596 & 629 & 1,710 & 2.72 \\
Subtotal & 288 & 3,031 & 3,319 & 8,676 & 2.73 \\
Total & 2,000 & 3,358 & 5,358 & 14,064 & 2.74 \\
\hline Note. IJHM = International Journal of Hospitality Management, JHTR = Journal of Hospitality and Tourism Research; CHQ = Cornell Hospitality \\
Quarterly, TM = Tourism Management, JTR = Journal of Travel Research, and ATR = Annals of Tourism Research
\end{tabular}

\section{Research Rankings in Hospitality}

The distributions of the research themes in hospitality during 2010-2019 are shown in Figure 1. Marketing and HRM got the most attention, while legal issues and education in the lodging industry gained the least attention.

Figure 1. Research Themes of Hospitality and Tourism During 2010-2019
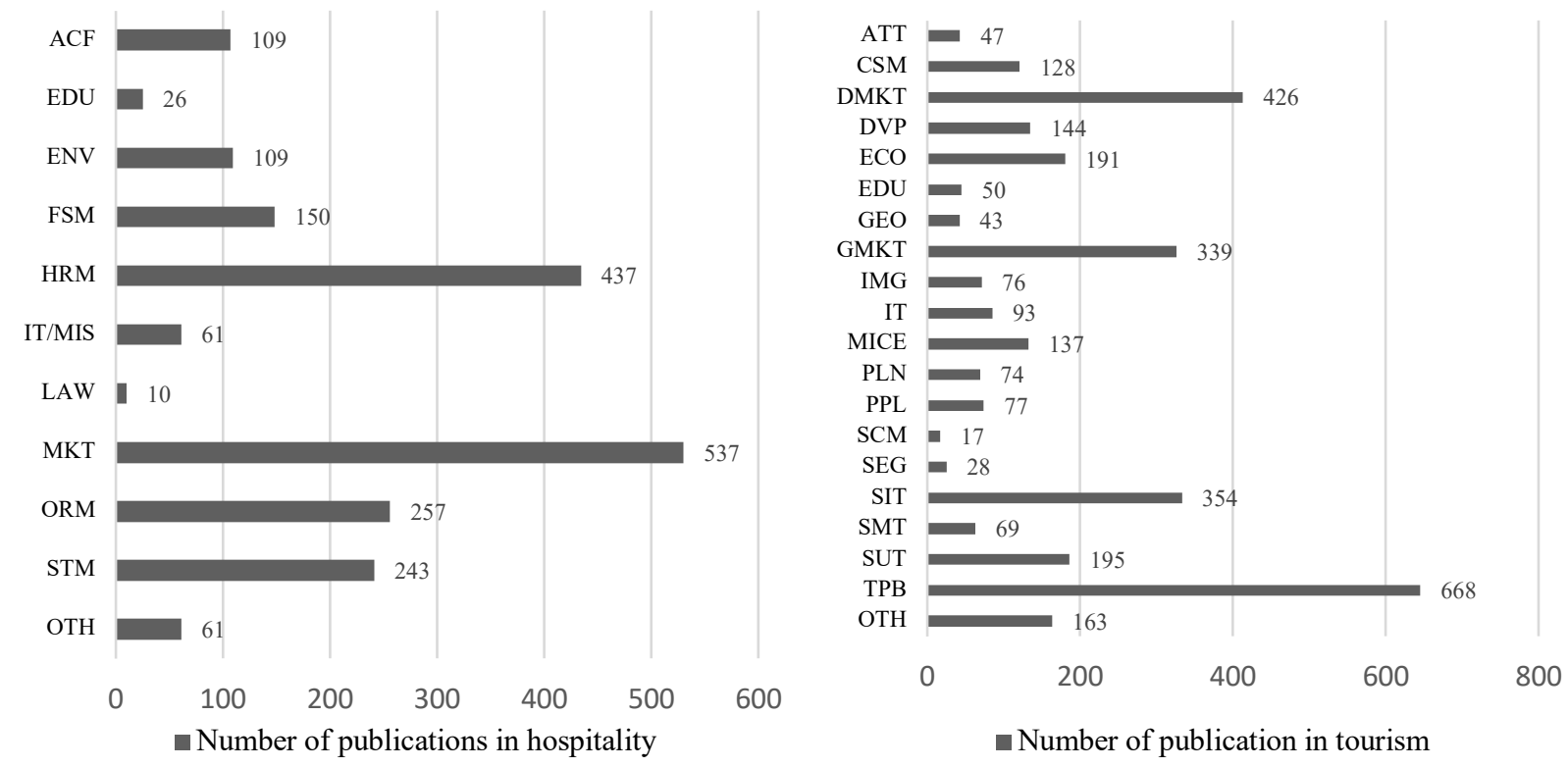

Note. $\mathrm{ACF}=$ Accounting and Finance; $\mathrm{EDU}=$ Education; $\mathrm{ENV}=$ Green And Environmental Issue; FSM = Food Service Management; HRM = Human Resource Management; IT/MIS = Information Technology and Management Information System; LAW = Legal Issue; MKT= Marketing; ORM = Operating Management; STM = Strategic Management; ATT $=$ Attraction Management; CSM = Crisis And Safety Management; DMKT $=$ Destination Marketing And Management; DVP = Tourism Development; ECO = Economic Impact and Econometrics; EDU = Education; GEO = Geographical Issue; GMKT = General Marketing; IMG = Image and Branding; IT = Information Technology; MICE = Meetings, Incentives, Conventions, and Exhibitions Including Festival and Fair; PLN = Tourism Planning; PPL = Politics, Policy, Legal, and Governmental Issue; SCM = Supply Chain

Management; SEG $=$ Segmentation; SIT $=$ Special Interests Tourism Such as Heritage, Farm, Cultural, Wine, or Food Tourism; SMT = Service Management; SUT = Sustainable

Tourism and Ecotourism; TPB = Tourists' Perception and Behavior; OTH $=$ Others 
As indicated in Table 2, the most productive 50 authors in hospitality published 804 papers with 357.56 points, accounting for $17.6 \%$ of the total points. In contrast, the top 50 authors during 2000 2009 gained 280.33 points at $25.9 \%$ of the total points (Park et al., 2011). SooCheong (Shawn) Jang, Ann S. Mattila, and Rob Law were among the top three from the perspectives of both score and number of publications. Specifically, SooCheong (Shawn) Jang published 69 papers with the highest score of 32.18. Ann S. Mattila, as the second, had 54 papers published with a score of 22.53. And the third was Rob Law, who published 43 papers with a score of 13.58. All these authors dominantly focused on marketing, while SooCheong (Shawn) Jang also did much work in strategical management. Differently, Anna S. Mattila, SooCheong (Shawn) Jang, and Cathy A. Enz ranked among the top three in terms of the score during 2000-2009.

Table 2. Author Ranking and Research Themes of Hospitality During 2010-2019

\begin{tabular}{|c|c|c|c|c|c|c|c|c|c|c|c|c|c|}
\hline Author Ranking by Score & Score & ACF & EDU & ENV & FSM & HRM & IT/MIS & LAW & MKT & ORM & STM & $\overline{\text { OTH }}$ & $\begin{array}{c}\text { Number of } \\
\text { Publication }\end{array}$ \\
\hline 1.SooCheong (Shawn) Jang & 32.18 & 4.33 & & 1.25 & 4.83 & 0.20 & & & 8.37 & 4.33 & 8.87 & & $69(1)$ \\
\hline 2.Anna S. Mattila & 22.53 & 0.25 & 0.20 & 0.33 & 1.50 & 1.08 & 1.33 & & 10.08 & 1.75 & 4.17 & 1.83 & $54(2)$ \\
\hline 3.Rob Law & 13.58 & & & & & 1.33 & 4.17 & & 4.83 & 1.42 & 0.50 & 1.33 & $43(3)$ \\
\hline 4.Seoki Lee & 12.87 & 3.25 & & 0.58 & 0.25 & 0.87 & 0.50 & & 0.75 & 3.75 & 2.92 & & $36(4)$ \\
\hline 5.Heesup Han & 12.07 & & & 3.08 & 0.87 & 1.00 & & & 5.28 & 0.25 & 1.08 & 0.50 & $33(5)$ \\
\hline 6.Ming-Hsiang Chen & 11.17 & 5.83 & & & & & & & 1.00 & 2.25 & 2.08 & & $17(13)$ \\
\hline 7.Juan M. Madera & 9.33 & & & & & 9.00 & & & 0.33 & & & & $19(8)$ \\
\hline 8.Manisha Singal & 9.00 & 3.92 & & 0.33 & & & & & 0.58 & 1.17 & 2.25 & 0.75 & $18(11)$ \\
\hline 9.Seul Ki Lee & 8.58 & 1.00 & & & 0.25 & & & & 2.00 & 2.00 & 3.33 & & $17(13)$ \\
\hline 10.Michael Lynn & 8.53 & & & & 2.20 & & & & 4.33 & 1.00 & 1.00 & & $13(22)$ \\
\hline 11.Li Miao & 8.40 & & & 1.00 & 1.08 & 0.75 & & & 1.28 & 0.75 & 1.20 & 2.33 & $21(7)$ \\
\hline 12.Alice H. Y. Hon & 7.83 & & & 0.17 & & 6.67 & & & 1.00 & & & & $14(20)$ \\
\hline 13.Sunghyup Sean Hyun & 7.58 & & & 0.50 & 0.50 & 0.33 & & & 4.92 & & 1.33 & & $16(17)$ \\
\hline 14.Woo Gon Kim & 7.48 & & & 0.83 & 0.92 & 2.90 & & & 1.67 & 0.83 & 0.33 & & $23(6)$ \\
\hline 15.Yang Yang & 7.08 & 0.50 & & & & & & & 1.67 & 2.33 & 2.25 & 0.33 & $19(8)$ \\
\hline 16. Hailin Qu & 6.81 & 0.33 & 0.33 & 0.14 & & 4.08 & & & 1.33 & 0.25 & 0.33 & & $19(8)$ \\
\hline 17.Lydia Hanks & 6.58 & & & 0.33 & 0.58 & 0.33 & & & 4.17 & 0.50 & 0.33 & 0.33 & $18(11)$ \\
\hline 18.A. George Assaf & 6.45 & & 0.25 & 0.33 & 0.25 & & & & 2.08 & 1.70 & 0.33 & 1.50 & $17(13)$ \\
\hline 19.Sarah Tanford & 6.33 & & & & & 1.00 & & & 3.67 & 0.67 & 1.00 & & $15(18)$ \\
\hline 20.Kwangmin Park & 6.17 & 1.33 & & & & & & & 0.83 & 1.00 & 3.00 & & $13(22)$ \\
\hline 21.John W. O'Neill & 6.08 & 1.50 & & & & 2.08 & & & & 1.50 & 0.50 & 0.50 & $11(30)$ \\
\hline 22.Lu Zhang & 5.67 & & & 0.33 & & & 0.33 & & 3.00 & & 1.67 & 0.33 & $13(22)$ \\
\hline 23.Dogan Gursoy & 5.58 & & & & & 2.00 & 1.33 & 0.33 & 1.58 & 0.33 & & & $13(22)$ \\
\hline 24.Anthony F. Lucas & 5.50 & & & & & & & & 2.00 & 3.50 & & & $8(44)$ \\
\hline 25.Zachary W. Brewster & 5.50 & & & & 1.00 & 1.50 & & & 0.50 & 1.50 & 0.50 & 0.50 & $8(44)$ \\
\hline 26.Hye Hyun Yoon & 5.33 & & & & 0.33 & 4.00 & & & 0.50 & 0.50 & & & $11(30)$ \\
\hline 26.Hyo Sun Jung & 5.33 & & & & 0.33 & 4.00 & & & 0.50 & 0.50 & & & $11(30)$ \\
\hline 26.Cristian Morosan & 5.33 & & & & & & 3.33 & & 2.00 & & & & $9(41)$ \\
\hline 29.Basak Denizci Guillet & 5.20 & 0.33 & & & & & 0.33 & & 2.03 & 0.92 & 1.33 & 0.25 & $15(18)$ \\
\hline 30.IpKin Anthony Wong & 5.12 & & & & & 1.12 & & & 1.50 & 1.50 & 1.00 & & $10(36)$ \\
\hline 31.Vincent P. Magnini & 4.92 & 0.50 & & & 0.33 & 0.67 & & & 2.33 & 0.33 & 0.75 & & $14(20)$ \\
\hline 32.Eric S. W. Chan & 4.78 & & & 1.50 & & 1.33 & & & 1.00 & 0.25 & 0.70 & & $10(36)$ \\
\hline 33.Dennis Reynolds & 4.73 & 0.33 & 0.40 & 1.17 & 1.25 & 0.25 & & & 0.83 & & 0.50 & & $13(22)$ \\
\hline 34.Nathaniel D. Line & 4.67 & & & 0.33 & 0.83 & 0.33 & & & 1.83 & 0.50 & 0.33 & 0.50 & $13(22)$ \\
\hline 35.Liang (Rebecca) Tang & 4.58 & & & & & & 0.33 & & 2.58 & & 1.67 & & $12(29)$ \\
\hline 36.Cindy Yoonjoung Heo & 4.50 & & & 0.25 & & & & & 0.83 & 1.83 & 1.58 & & $9(41)$ \\
\hline 36.Donghee Kim & 4.50 & & & & 1.00 & & & & 3.00 & & 0.50 & & $9(41)$ \\
\hline 36.Amrik Singh & 4.50 & 4.00 & & & & & & & 0.50 & & & & $5(48)$ \\
\hline 39.Hyun Jeong Kim & 4.42 & & & 0.33 & & 1.67 & & & 1.58 & 0.50 & & 0.33 & $11(30)$ \\
\hline 40.Alex M. Susskind & 4.37 & & & 1.00 & 2.33 & 0.20 & & & 0.50 & 0.33 & & & $7(46)$ \\
\hline 41.Fevzi Okumus & 4.33 & 0.20 & & & & 1.17 & & & 0.45 & 0.75 & 1.57 & 0.20 & $17(13)$ \\
\hline 41.Priyanko Guchait & 4.33 & & & & 0.33 & 2.33 & & & 0.50 & 0.33 & 0.83 & & $11(30)$ \\
\hline 41.Osman M. Karatepe & 4.33 & & & & & 4.33 & & & & & & & $7(46)$ \\
\hline 44.Levent Altinay & 4.20 & & 0.25 & & & 0.33 & & & 1.25 & 1.83 & 0.53 & & $11(30)$ \\
\hline 45.Xinyuan (Roy) Zhao & 4.09 & 0.25 & & 0.14 & & 2.58 & & & 0.53 & 0.58 & & & $13(22)$ \\
\hline 46.Céline Jacob & 4.08 & & & & 1.75 & 0.33 & & & 1.00 & 0.50 & 0.50 & & $10(36)$ \\
\hline 46.Nicolas Guéguen & 4.08 & & & & 1.75 & 0.33 & & & 1.00 & 0.50 & 0.50 & & $10(36)$ \\
\hline 46.Chiang-Ming Chen & 4.08 & 0.50 & & & & 0.25 & & & 1.58 & 1.00 & 0.75 & & $10(36)$ \\
\hline 49.Rajib Lochan Dhar & 4.00 & & & 0.50 & & 3.50 & & & & & & & $5(48)$ \\
\hline 49.Gary M. Thompson & 4.00 & 1.00 & & & & & & & & 3.00 & & & $4(50)$ \\
\hline
\end{tabular}

Note. ACF = Accounting and Finance; EDU = Education; ENV = Green and Environmental Issue; FSM = Food Service Management; HRM = Human Resource Management; IT/MIS = Information Technology and Management Information System; LAW = Legal Issue; MKT = Marketing; ORM = Operating Management; STM = Strategic Management; $\mathrm{OTH}=$ Others. In the column of number of publications, the first number is the count of papers, and the second number in parentheses is the ranking based on the count of papers.

As indicated in Table 3, the top 50 universities gained 1053.54 points, accounting for $52.7 \%$ of the total points for hospitality research during 2010-2019. The results were similar to those during 2000-2009 (i.e., 702.69 points; 64.9\%). The top three universities by both score and number of publications were Hong Kong Polytechnic University (i.e., 111.73 points; 181 papers), 
Pennsylvania State University (i.e., 75.23 points; 123 papers), and Purdue University (i.e., 70.03 points, 103 papers). The most research efficient university by weighted score was Pennsylvania State University (i.e., 5.01 points). All of the three universities emphasized on marketing and strategy management. Both Hong Kong Polytechnic University and Pennsylvania State University also focused on HRM and operation management. Purdue University addressed food service management as well. Differently, during 2000-2009 the three most prolific universities were Cornell University, Hong Kong Polytechnic University, and Pennsylvania State University, while the most research efficient university by weighted score was Oxford Brookes University.

Table 3. University Ranking and Research Themes of Hospitality During 2010-2019

\begin{tabular}{|c|c|c|c|c|c|c|c|c|c|c|c|c|c|c|}
\hline University Ranking by Score & Score & ACF & EDU & ENV & FSM & HRM & IT/MIS & LAW & MKT & ORM & STM & OTH & $\begin{array}{r}\text { Weighted } \\
\text { Score }\end{array}$ & $\begin{array}{c}\text { Number of } \\
\text { Publication }\end{array}$ \\
\hline 1.HK Poly U. & 111.73 & 3.40 & 0.50 & 7.38 & 2.33 & 27.45 & 8.92 & 1.00 & 28.78 & 12.92 & 10.32 & 8.73 & $2.06(8)$ & 181(1) \\
\hline 2.Pennsylvania State University & 75.23 & 5.17 & 0.20 & 1.00 & 6.75 & 10.45 & 2.33 & & 23.75 & 11.25 & 11.67 & 2.67 & $5.01(1)$ & $123(2)$ \\
\hline 3.Purdue University & 70.03 & 6.33 & 1.25 & 2.75 & 11.50 & 2.13 & & & 19.42 & 8.18 & 14.80 & 3.67 & $3.50(4)$ & 103(3) \\
\hline 4.Cornell University & 66.38 & 9.17 & 2.33 & 4.18 & 7.50 & 7.62 & & 1.00 & 14.33 & 12.58 & 6.67 & 1.00 & $1.84(11)$ & $98(4)$ \\
\hline 5.University of Central Florida & 50.33 & 1.87 & & 1.33 & 6.57 & 10.85 & 1.33 & & 10.53 & 8.25 & 7.07 & 2.53 & $0.88(31)$ & $91(5)$ \\
\hline 6.University of Houston & 42.78 & 1.92 & 0.25 & 0.50 & 1.83 & 20.92 & 4.67 & & 9.50 & 2.37 & 0.83 & & $1.86(9)$ & $62(8)$ \\
\hline 7.Florida State University & 38.53 & 1.50 & & 3.83 & 5.12 & 10.92 & & & 11.00 & 4.00 & 2.17 & & $3.85(2)$ & $57(10)$ \\
\hline 7.Virginia Tech & 38.33 & 7.00 & & 1.67 & 1.42 & 5.75 & 2.33 & & 10.58 & 4.17 & 3.17 & 2.25 & $2.45(5)$ & $65(7)$ \\
\hline 9.University of Nevada, Las Vegas & 37.87 & 0.50 & & 1.50 & 1.75 & 8.78 & 0.33 & & 14.50 & 6.17 & 3.67 & 0.67 & $1.14(26)$ & $60(9)$ \\
\hline 10.Kyung Hee University & 33.90 & 1.83 & & 1.25 & 2.75 & 13.23 & 1.00 & & 9.42 & 2.92 & 1.50 & & $1.21(24)$ & $55(12)$ \\
\hline 11.Temple University & 33.58 & 4.75 & & 0.75 & 0.75 & 4.67 & 1.17 & & 8.08 & 4.83 & 8.25 & 0.33 & $1.15(25)$ & $69(6)$ \\
\hline 12. Washington State University & 28.57 & 3.17 & 0.20 & 3.33 & 2.17 & 6.25 & 1.33 & 0.67 & 7.77 & 1.58 & 1.43 & 0.67 & $1.78(13)$ & $48(14)$ \\
\hline 13.Sun Yat-Sen University & 27.41 & 1.25 & & 0.86 & 2.50 & 9.88 & 0.33 & & 5.17 & 3.50 & 3.25 & 0.67 & 1.71(16) & $55(12)$ \\
\hline 14. Sejong University & 25.48 & 1.33 & & 2.50 & 1.45 & 2.00 & 0.33 & & 8.78 & 2.67 & 6.42 & & $0.82(33)$ & $56(11)$ \\
\hline 15.Griffith University & 24.32 & 2.50 & 0.50 & 1.25 & 2.00 & 6.15 & & & 7.50 & 2.25 & 0.67 & 1.50 & $0.65(35)$ & $39(16)$ \\
\hline 16.University of Massachusetts & 20.40 & & 1.08 & 0.67 & 1.08 & 1.17 & 1.00 & 1.00 & 8.33 & 2.57 & 1.67 & 1.83 & $1.36(20)$ & $41(15)$ \\
\hline 17.Michigan State University & 18.52 & 2.00 & 0.50 & 0.33 & 0.33 & 1.93 & 0.33 & & 8.33 & 2.08 & 2.67 & & $1.85(10)$ & $30(18)$ \\
\hline 18.Oklahoma State University & 18.45 & 1.50 & 0.33 & 0.50 & 1.70 & 6.58 & & & 4.17 & 1.00 & 2.67 & & $2.30(7)$ & $39(16)$ \\
\hline 19.Iowa State University & 17.25 & & 1.00 & 1.00 & 2.58 & 2.83 & 0.67 & & 5.50 & & 3.67 & & $1.56(18)$ & $28(19)$ \\
\hline 20.University of Macau & 14.63 & 1.25 & & & & 6.50 & & 1.00 & 5.13 & 0.50 & 0.25 & & $1.12(27)$ & $21(24)$ \\
\hline 21. Oxford Brookes University & 14.62 & & 0.75 & 0.50 & 1.25 & 1.17 & & & 2.08 & 7.00 & 0.87 & 1.00 & $3.65(3)$ & $24(22)$ \\
\hline 22. University of South Carolina & 12.12 & 0.50 & 0.25 & & 2.50 & 1.08 & & 0.67 & 5.45 & 0.67 & 1.00 & & $0.35(41)$ & $25(20)$ \\
\hline 23.University of Queensland & 12.05 & 1.00 & 0.20 & 0.33 & 0.50 & 2.77 & 1.00 & & 2.67 & 1.83 & 0.75 & 1.00 & $1.72(15)$ & $21(24)$ \\
\hline 24.Ohio State University & 12.02 & & 0.20 & 0.33 & 1.50 & 3.50 & 1.00 & & 3.95 & 0.33 & 1.20 & & $2.40(6)$ & $25(21)$ \\
\hline 25.ULPGC & 11.67 & & & 0.67 & & 3.00 & 1.00 & & 3.50 & 3.00 & 0.50 & & $\mathrm{n} / \mathrm{a}$ & $14(31)$ \\
\hline 26.Bournemouth University & 11.00 & & & & 2.00 & 2.92 & 0.50 & & 1.08 & 1.33 & 2.17 & 1.00 & $0.31(42)$ & $19(26)$ \\
\hline 27.Ming Chuan University & 9.93 & & & 1.48 & 1.00 & 2.28 & 0.33 & & 3.83 & & 1.00 & & $1.24(23)$ & $19(26)$ \\
\hline 28.Dong-A University & 9.53 & 1.00 & & 1.33 & 1.37 & & & & 3.92 & & 1.42 & 0.50 & $0.86(32)$ & $22(23)$ \\
\hline 29.University of South Australia & 9.45 & & & & 1.92 & 3.00 & & & 1.53 & 3.00 & & & $1.57(17)$ & $14(31)$ \\
\hline 30.National Chi Nan University & 9.00 & 1.00 & & & & 2.83 & & & 2.83 & 1.00 & 1.08 & 0.25 & $0.64(36)$ & $9(47)$ \\
\hline 30.University of Alicante & 9.00 & & & 2.00 & & 2.00 & & & 1.00 & 2.00 & 1.00 & 1.00 & $\mathrm{n} / \mathrm{a}$ & $16(30)$ \\
\hline 32.University of Denver & 8.95 & 4.00 & & & 0.25 & 0.50 & 0.50 & & 2.37 & & 1.33 & & $1.79(12)$ & $17(29)$ \\
\hline 33.Université de Bretagne-Sud & 8.83 & & & & 3.83 & 1.00 & & & 2.00 & 1.00 & 1.00 & & $\mathrm{n} / \mathrm{a}$ & $10(45)$ \\
\hline 34.Ecole Hoteliere de Lausanne & 8.67 & & & & 1.00 & 2.17 & 2.00 & & 2.00 & & 1.50 & & $0.29(43)$ & $12(39)$ \\
\hline 35.Kansas State University & 8.45 & & & 1.50 & 3.17 & 2.58 & & & 1.20 & & & & $1.40(19)$ & 13(36) \\
\hline 36.E. Mediterranean & 7.58 & & & & & 7.58 & & & & & & & $0.64(37)$ & $10(45)$ \\
\hline 36. National Chung Cheng & 7.58 & 3.33 & & & & & & & 1.00 & 1.75 & 1.50 & & $\mathrm{n} / \mathrm{a}$ & $9(47)$ \\
\hline 38.HES-SO & 7.50 & 1.00 & & & & 2.08 & & & 1.92 & 1.00 & 1.50 & & $\mathrm{n} / \mathrm{a}$ & $12(39)$ \\
\hline 39. University of Delaware & 7.42 & & & & & & 1.00 & & 3.58 & 1.67 & 1.17 & & $0.57(38)$ & $12(39)$ \\
\hline 40.Universidad de Málaga & 7.25 & 1.00 & & & & 3.75 & & & 0.50 & 1.00 & 1.00 & & $\mathrm{n} / \mathrm{a}$ & $8(50)$ \\
\hline 41.California State Polytechnic University & 7.17 & 0.75 & & 1.50 & 0.58 & & 0.33 & & 2.83 & 0.33 & 0.50 & 0.33 & $1.02(29)$ & $19(26)$ \\
\hline 41.National Chiayi University & 7.17 & & & & & 5.00 & & & 1.83 & & 0.33 & & $0.39(40)$ & $14(31)$ \\
\hline 43.University of Missouri & 7.00 & 0.50 & 0.33 & 1.00 & 1.00 & 1.33 & & & 1.17 & 1.67 & & & $1.75(14)$ & $12(39)$ \\
\hline 44.Florida International University & 6.92 & & & 0.33 & 1.00 & & & & 3.92 & 0.67 & 1.00 & & $0.25(44)$ & $13(36)$ \\
\hline 45.Texas Tech University & 6.83 & & 1.33 & & 0.92 & 0.25 & 0.33 & & 1.00 & 1.00 & 2.00 & & $0.48(39)$ & $14(31)$ \\
\hline 46.Hong Kong Baptist University & 6.75 & & & & & 4.75 & & & 1.00 & 1.00 & & & $1.35(21)$ & $11(44)$ \\
\hline 47.University of Florida & 6.58 & & & & 1.00 & 0.25 & & & 2.50 & 0.75 & 2.08 & & $0.94(30)$ & $13(38)$ \\
\hline 48.Pusan National University & 6.33 & & & & 0.50 & & & & 3.17 & 0.83 & 1.83 & & $1.26(22)$ & $12(39)$ \\
\hline 49. University of New Hampshire & 6.25 & 1.00 & & 1.50 & 1.17 & 2.00 & & & 0.58 & & & & $1.04(28)$ & $9(47)$ \\
\hline 50.University of Surrey & 6.20 & & & & 0.75 & 1.62 & & & 1.58 & 1.25 & 1.00 & & $0.68(34)$ & $14(31)$ \\
\hline
\end{tabular}

Note. $\mathrm{ACF}=$ Accounting and Finance; EDU = Education; ENV = Green and Environmental Issue; FSM = Food Service Management; HRM = Human Resource Management;

IT/MIS = Information Technology and Management Information System; LAW = Legal Issue; MKT = Marketing; ORM = Operating Management; STM = Strategic Management; OTH = Others; HK Poly U.= The Hong Kong Polytechnic University; Virginia Tech = Virginia Polytechnic Institute and State University; ULPGC = Universidad de Las Palmas de Gran Canaria; E. Mediterranean = Eastern Mediterranean University; HES-SO = HES-SO University of Applied Sciences and Arts Western Switzerland. In the column of number of publications, the first number is the count of papers, and the second number in parentheses is the ranking based on the count of papers. The same pattern is shown in the column of weighted score.

The top 20 countries/regions by the score in hospitality research during 2010-2019 are demonstrated in Table 4. The top three countries/regions were the United States (i.e., 853.48 points, 42.7\%), Taiwan (i.e., 150.50 points, 30.1\%), and Hong Kong (i.e., 129.78 points, $25.9 \%$ ). However, during 2000-2009 the top three countries/regions by score were the United States, Hong Kong, and the United Kingdom. 
Table 4. Country/Region Ranking and Research Areas of Hospitality During 2010-2019

\begin{tabular}{|c|c|c|c|c|c|c|c|c|c|c|c|c|c|}
\hline $\begin{array}{l}\text { Country/Region } \\
\text { Ranking by Score }\end{array}$ & Score & ACF & EDU & ENV & FSM & HRM & IT/MIS & LAW & MKT & ORM & STM & OTH & $\begin{array}{l}\text { Number of } \\
\text { Publication }\end{array}$ \\
\hline 1.USA & 853.48 & 58.28 & 16.23 & 36.68 & 83.15 & 149.23 & 30.83 & 5.67 & 246.37 & 101.57 & 103.02 & 22.45 & $1022(1)$ \\
\hline 2.Taiwan & 150.50 & 5.67 & 0.75 & 15.92 & 8.92 & 47.25 & 2.08 & & 32.00 & 15.08 & 21.58 & 1.25 & $171(5)$ \\
\hline 3.Hong Kong & 129.78 & 3.40 & 1.83 & 8.25 & 2.83 & 35.30 & 9.42 & 1.00 & 32.45 & 14.42 & 11.65 & 9.23 & $206(2)$ \\
\hline 4.Korea & 114.48 & 4.17 & & 8.17 & 13.22 & 21.88 & 3.00 & & 38.22 & 10.42 & 14.92 & 0.50 & $178(4)$ \\
\hline 5.Spain & 108.53 & 8.50 & & 8.57 & 1.00 & 22.75 & 3.00 & & 25.42 & 16.17 & 19.18 & 3.75 & $122(8)$ \\
\hline 6.Mainland China & 103.20 & 3.58 & 0.58 & 3.08 & 4.37 & 37.78 & 3.33 & & 25.13 & 12.00 & 11.33 & 2.00 & $180(3)$ \\
\hline 7.UK & 87.35 & 1.50 & 2.50 & 3.50 & 6.17 & 20.03 & 1.17 & & 19.23 & 18.62 & 9.03 & 5.00 & $141(6)$ \\
\hline 8.Australia & 86.15 & 3.75 & 2.40 & 4.72 & 8.50 & 21.67 & 2.00 & & 19.55 & 13.32 & 4.75 & 5.50 & $125(7)$ \\
\hline 9.Macao & 35.32 & 1.25 & & & 1.00 & 16.60 & & 1.00 & 10.80 & 2.00 & 2.42 & 0.25 & 49(9) \\
\hline 10.Italy & 32.43 & 3.00 & & 2.75 & 3.75 & & & & 6.78 & 7.08 & 6.67 & 2.20 & $45(11)$ \\
\hline 11.Turkey & 30.13 & 1.73 & & 0.25 & & 11.67 & & & 3.20 & 3.67 & 7.17 & 2.45 & $46(10)$ \\
\hline 12.Switzerland & 25.20 & 2.00 & & & 1.00 & 5.28 & 2.00 & & 5.17 & 4.00 & 5.75 & & $39(12)$ \\
\hline 13.India & 19.48 & 1.00 & & 3.00 & & 4.73 & & & 6.75 & 3.17 & 0.83 & & $25(15)$ \\
\hline 14.France & 18.75 & & & 1.00 & 6.00 & 2.00 & 1.00 & & 4.75 & 2.50 & 1.50 & & $24(14)$ \\
\hline 15.Portugal & 18.75 & 2.67 & & 1.00 & & 1.33 & & & 7.75 & 4.00 & 1.33 & 0.67 & $26(16)$ \\
\hline 16.New Zealand & 18.27 & & 0.20 & 0.25 & 1.40 & 5.83 & & 1.33 & 4.00 & 2.00 & 2.50 & 0.75 & $30(13)$ \\
\hline 17.Israel & 13.92 & & & & 1.00 & 4.00 & 1.00 & 1.00 & 3.33 & 1.33 & & 2.25 & $16(19)$ \\
\hline 18.Malaysia & 13.27 & & & 0.67 & 1.20 & 4.03 & & & 4.03 & 3.00 & 0.33 & & $20(17)$ \\
\hline 19.Canada & 10.50 & 0.50 & & 0.58 & 1.00 & 0.50 & & & 4.92 & 1.67 & 1.33 & & $17(18)$ \\
\hline 20.Austria & 10.08 & 1.00 & & 1.33 & & 1.67 & 0.33 & & 1.67 & 4.08 & & & $15(20)$ \\
\hline
\end{tabular}

\section{Research Rankings in Tourism}

Figure 1 demonstrates the summary of tourism research themes during 2010-2019. The dominant themes included general tourism, special interest tourism, general marketing, and destination marketing, while the themes with the least attention were tourism segmentation and supply chain management. Table 5 shows the top 50 authors in tourism during 2010-2019. These 50 authors published 849 papers with 357.13 points, which was $10.6 \%$ of the total points for tourism research. In contrast, the total score of the top 50 authors was 306.36 , which was $17.5 \%$ of the total points for tourism papers during 2000-2009. The top three authors by the score were Rob Law (14.65 points, 46 papers), Xiang (Robert), Li (13.85 points, 37 papers), and Kyle M. Woosnam (13.10 points, 30 papers). However, the top three authors by the number of papers were Rob Law, Haiyan Song, and Xiang (Robert) Li. Both Rob Law and Kyle M. Woosnam focused on tourists' perceptions and behaviors, and Haiyan Song addressed economic impact and econometrics, while Xiang (Robert) Li mostly gave attention to general marketing and tourists' perceptions and behaviors.

Table 6 shows the top 50 universities in tourism during 2010-2019. The top 50 universities got 1277.88 points at $38.1 \%$ of the total points of the tourism papers, compared to $45.1 \%$ during 2000 2009. During 2010-2019 the top three universities by the score were Hong Kong Polytechnic University (144.68 points, 4.3\%), Griffith University (80.62 points, $2.4 \%$ ), and University of Queensland (70.88 points, 2.1\%). All of the three universities focused on tourists' perceptions and behaviors. Besides, Hong Kong Polytechnic University gave attention to general marketing and destination marketing, and Griffith University addressed sustainable tourism as well. In contrast, during 2000-2009, the top three universities by the score were Hong Kong Polytechnic University, Texas A\&M University, and Griffith University. In terms of weighted score, the most prolific university was Southern Cross University during 2010-2019, while it was the University of Nottingham during 2000-2009. 
Table 5. Author Ranking and Research Themes of Tourism During 2010-2019

\begin{tabular}{|c|c|c|c|c|c|c|c|c|c|c|c|c|c|c|c|c|c|c|c|c|c|c|}
\hline Author Ranking by Score & Score & ATT & CSM & DMKT & DVP & ECO & EDU & GEO & GMKT & IMG & IT & MICE & PLN & PPL & SCM & SEG & SIT & SMT & SUT & TPB & OTH & $\begin{array}{c}\text { Number of } \\
\text { Publication }\end{array}$ \\
\hline 1.Rob Law & 14.65 & & 0.33 & 1.92 & 0.25 & 1.00 & & & 1.73 & & 1.67 & & 0.92 & & & & & & & 3.25 & 3.58 & $46(1)$ \\
\hline 2.Xiang (Robert) Li & 13.85 & 0.58 & & 3.08 & & & & & 1.78 & 1.70 & 0.25 & 0.67 & & & & & 1.33 & & & 3.37 & 1.08 & $37(3)$ \\
\hline 3.Kyle M. Woosnam & 13.10 & 0.25 & & 0.67 & 0.40 & 0.58 & & & 0.67 & & 1.00 & 0.50 & & & & & 1.00 & 1.00 & 0.25 & 2.78 & 4.00 & $30(4)$ \\
\hline 4.Haiyan Song & 13.03 & & 0.58 & 1.42 & 1.83 & 3.53 & & & 1.58 & 0.50 & 0.25 & 0.25 & 1.00 & 0.33 & 0.58 & & & 0.33 & & 0.25 & 0.58 & $39(2)$ \\
\hline 5.Bob McKercher & 12.28 & 0.50 & & 1.42 & 0.33 & & 0.25 & & 1.53 & & 0.50 & & 0.50 & 0.50 & & 1.00 & 0.50 & & & 1.75 & 3.50 & $25(9)$ \\
\hline 6.Sara Dolnicar & 11.58 & & 0.92 & 1.25 & & & & & 3.50 & & & & & & & 0.58 & & & 1.17 & 2.33 & 1.83 & $30(4)$ \\
\hline 7.A. George Assaf & 11.03 & & & 2.58 & & & & 1.33 & 1.17 & 0.25 & 0.50 & & 0.50 & & & & & & & 2.53 & 2.17 & $30(4)$ \\
\hline 8.Chris Ryan & 10.42 & & & 1.83 & 1.00 & & 0.33 & & 0.58 & & & 0.50 & 0.33 & & & & 3.00 & & & 2.00 & 0.83 & $26(8)$ \\
\hline 9.Ralf Buckley & 9.70 & 0.50 & 2.00 & 0.20 & & & & & 0.33 & & & & & & & & 1.83 & & 2.00 & 1.33 & 1.50 & $14(27)$ \\
\hline 10.Juan L. Nicolau & 9.25 & & & 1.83 & & 0.83 & & & 0.50 & 1.00 & & 0.50 & & & & 1.00 & & & & 3.58 & & $17(17)$ \\
\hline 11.James F. Petrick & 8.92 & 1.00 & & 0.33 & & 0.50 & 0.50 & & 1.83 & 0.75 & & & & & & & 0.25 & & & 2.50 & 1.25 & $22(12)$ \\
\hline 12.SooCheong (Shawn) Jang & 8.87 & 0.50 & 1.00 & 2.03 & & 1.25 & & & 1.33 & & & 0.50 & 0.25 & & & & 0.50 & & & 1.00 & 0.50 & $19(14)$ \\
\hline 13.David B. Weaver & 8.42 & & & & & & & & 0.33 & & & 0.50 & 0.33 & 0.33 & & 0.33 & 1.00 & & 5.33 & & 0.25 & $14(27)$ \\
\hline 14.Gang Li & 8.32 & & & 1.08 & 0.58 & 1.20 & & & 1.70 & & 1.00 & & 0.33 & & & & & & & 1.00 & 1.42 & $29(7)$ \\
\hline 15.Heesup Han & 7.95 & & & 0.25 & & & & & 1.50 & 0.50 & & & & & & & 1.50 & & & 4.20 & & $16(20)$ \\
\hline 16. Muchazondida Mkono & 7.83 & & & & & & & & 1.00 & & 1.50 & & & & & & & & & 4.00 & 1.33 & $10(39)$ \\
\hline 17.Dogan Gursoy & 7.78 & & & 0.83 & 0.83 & & & & 1.00 & & & 0.70 & & 0.67 & & & & & 0.33 & 2.92 & 0.50 & $24(10)$ \\
\hline 18. Susanne Becken & 7.42 & & 2.00 & 1.50 & & & & & & & 0.33 & & & & & 0.50 & & & 0.92 & 2.17 & & $15(21)$ \\
\hline 19.Songshan (Sam) Huang & 7.07 & 0.53 & & 0.33 & 0.33 & 0.83 & & & 0.33 & & & & & & & & 0.33 & & & 3.37 & 1.00 & $18(16)$ \\
\hline 20.Svetlana Stepchenkova & 6.95 & 0.33 & 0.33 & 2.87 & & & & & 0.83 & 1.00 & 0.58 & 0.50 & & & & & & & & 0.50 & & $17(17)$ \\
\hline 21.Robin Nunkoo & 6.75 & & & 0.50 & 0.33 & 0.50 & & & & & & 0.50 & & 2.33 & & & 0.83 & & & 1.75 & & $15(21)$ \\
\hline 22.Brent Ritchie & 6.73 & 0.20 & 2.00 & 0.25 & 0.17 & 0.25 & & & 0.25 & & & 0.58 & & & & & 1.00 & 0.20 & & 1.08 & 0.75 & $23(11)$ \\
\hline 23.Philip L. Pearce & 6.37 & 0.50 & & & & & & & 1.50 & & & & & & & & 0.33 & 0.50 & & 3.53 & & $12(32)$ \\
\hline 24.Yang Yang & 6.33 & & & 0.67 & & 0.83 & & 0.50 & 0.75 & & 0.50 & & & & 0.50 & & 1.00 & & & 1.25 & 0.33 & $15(21)$ \\
\hline 25.Choong-Ki Lee & 6.32 & & & 0.33 & & & & & 0.58 & & & 1.25 & & & & & 0.50 & 0.25 & & 3.40 & & $17(17)$ \\
\hline 26.Kam Hung & 6.28 & & & 0.58 & & & & & 1.00 & 0.50 & & & & & & & 0.50 & & & 2.20 & 1.50 & $22(12)$ \\
\hline 27.Bing Pan & 6.12 & & & 3.00 & 0.25 & & & & & 0.20 & 1.08 & & 0.25 & & & & & & & 1.00 & 0.33 & $14(27)$ \\
\hline 28.Larry Dwyer & 6.03 & & & 1.78 & & 1.67 & & 0.67 & 0.33 & & & & & 1.00 & & & 0.25 & & & 0.33 & & $19(14)$ \\
\hline 29.John Tribe & 5.95 & & & & & & 0.25 & & 1.00 & & & & 0.33 & & & & 0.33 & & 0.20 & 0.33 & 3.50 & $13(32)$ \\
\hline 30.IpKin Anthony Wong & 5.62 & & & 1.50 & 0.33 & & 0.33 & & 0.20 & & & 0.67 & & & & & 1.50 & & & 1.08 & & $15(21)$ \\
\hline 31.Konstantinos Andriotis & 5.50 & & 1.00 & & & & & & & 0.50 & & & & & & & 2.00 & & & 1.50 & 0.50 & $7(46)$ \\
\hline 32.Bill Bramwell & 5.33 & & & & 1.50 & 0.50 & & & & & & & & 1.50 & & & 0.33 & & 1.00 & & 0.50 & $10(39)$ \\
\hline 33.Sangwon Park & 5.17 & & 0.33 & 1.17 & & & & & 1.50 & 0.50 & & & & & & & 0.33 & & & 1.00 & 0.33 & $12(34)$ \\
\hline 34.Daniel R. Fesenmaier & 5.08 & & & 2.58 & & & & & 0.50 & & 0.67 & & 0.25 & & & & & & & 0.75 & 0.33 & $14(27)$ \\
\hline 35. Honggang Xu & 4.92 & & & 1.58 & 1.00 & & & & 0.67 & & & & & 0.50 & & & 0.33 & & & 0.83 & & $14(27)$ \\
\hline 35.Scott McCabe & 4.92 & & & 0.58 & & 0.33 & & & 1.08 & & & & & & & & 0.33 & & & 2.58 & & $12(34)$ \\
\hline 37.Nancy Gard McGehee & 4.87 & & & 0.25 & 0.33 & & & 0.17 & & & & & & 0.25 & & & 1.83 & & 0.83 & 0.95 & 0.25 & $13(32)$ \\
\hline 38.Ching-Fu Chen & 4.83 & & & 0.50 & & 0.33 & 0.50 & & & & & & & & & & 0.50 & 1.00 & & 2.00 & & $11(37)$ \\
\hline 38.Sheng-Hshiung Tsaur & 4.83 & & & & 0.33 & & 0.83 & & 0.50 & & & & & & & & 0.33 & 1.00 & & 1.33 & 0.50 & $10(39)$ \\
\hline 40.B. Bynum Boley & 4.78 & & & 0.17 & & 0.33 & & & 0.33 & & 0.33 & & 0.50 & & & & & & 1.75 & 1.37 & & $15(21)$ \\
\hline 41.Iis P. Tussyadiah & 4.75 & 0.33 & & 0.58 & & & & 0.50 & 1.00 & & 0.50 & & 1.00 & & & & & & & 0.83 & & $10(39)$ \\
\hline 41.Dallen J. Timothy & 4.75 & & & & 0.50 & & 1.00 & & 0.33 & & & & & 0.50 & & & 1.83 & & & 0.58 & & $10(39)$ \\
\hline 43.Egon Smeral & 4.67 & & & 0.33 & & 2.00 & & & 0.33 & & & & & & & & & & & 1.00 & 1.00 & $6(49)$ \\
\hline 44.Nigel Morgan & 4.62 & 0.33 & & 0.25 & 0.33 & 0.33 & & & & & & & 0.33 & & & & 1.25 & & & 1.00 & 0.78 & $15(21)$ \\
\hline ichainuwat & 4.58 & & 2.00 & & & & & & & & & 0.50 & & & & & 0.75 & & & 1.33 & & $7(46)$ \\
\hline 45.Ming-Hsiang Chen & 4.58 & & 1.00 & & 1.00 & 2.00 & & & 0.33 & & & & & & & & & & & 0.25 & & 6(49) \\
\hline 47.Robertico Croes & 4.53 & & & 2.03 & 1.00 & 0.83 & & & 0.67 & & & & & & & & & & & & & $9(45)$ \\
\hline 48.Gyan P. Nyaupane & 4.50 & & & 1.00 & & & & & & & & & & 0.50 & & & 1.17 & & 0.83 & 0.50 & 0.50 & $11(37)$ \\
\hline 48.Honggen Xiao & 4.50 & & & & 0.50 & 0.67 & 0.25 & & & & & & & & & & 0.25 & & & 1.00 & 1.83 & $10(39)$ \\
\hline 48.Ya-Yen Sun & 4.50 & & & 0.25 & & & & & & & & 0.25 & & & & & & & 4.00 & & & $7(46)$ \\
\hline
\end{tabular}

Note. ATT = Attraction Management; CSM = Crisis and Safety Management; DMKT = Destination Marketing and Management; DVP = Tourism Development; ECO = Economic Impact and Econometrics; EDU = Education; GEO = Geographical Issue; GMKT = General Marketing; IMG = Image and Branding; IT = Information Technology; MICE = Meetings, Incentives, Conventions, and Exhibitions Including Festival and Fair; PLN = Tourism Planning; PPL = Politics, Policy, Legal, and Governmental Issue; SCM = Supply Chain Management; SEG = Segmentation; SIT = Special Interests Tourism such as Heritage, Farm, Cultural, Wine, or Food Tourism; SMT = Service Management; SUT = Sustainable Tourism and Ecotourism; TPB = Tourists' Perception and Behavior; OTH = Others; U. = University; UCF = University of Central Florida; B-GUN = Ben-Gurion University of the Negev; UNLV = University of Nevada, Las Vegas; ULPGC = University of Las Palmas de Grad Canaria. In the column of number of publications, the first number is the count of papers, and the second number in parentheses is the ranking based on the count of papers. 
Table 6. University Ranking and Research Themes of Tourism During 2010-2019

\begin{tabular}{|c|c|c|c|c|c|c|c|c|c|c|c|c|c|c|c|c|c|c|c|c|c|c|c|}
\hline $\begin{array}{l}\text { University Ranking by } \\
\text { Score }\end{array}$ & Score & ATT & CSM & DMKT & DVP & ECO & EDU & GEO & GMKT & IMG & IT & MICE & PLN & PPL & SCM & SEG & $\begin{array}{c}\text { SIT } \\
\end{array}$ & SMT & SUT & TPB & OTH & $\begin{array}{r}\text { Weighted } \\
\text { Score }\end{array}$ & $\begin{array}{r}\begin{array}{r}\text { Number of } \\
\text { Publications }\end{array} \\
\text { rom }\end{array}$ \\
\hline 1.HK Poly U. & 144.68 & 1.17 & 3.75 & 18.25 & 5.08 & 8.20 & 2.00 & 0.67 & 17.88 & 5.67 & 5.50 & 6.42 & 4.08 & 4.75 & 0.58 & 1.00 & 7.83 & 1.50 & 1.32 & 33.28 & 15.75 & $2.67(10)$ & $264(1)$ \\
\hline 2.Griffith University & $\begin{array}{l}80.62 \\
70.88\end{array}$ & 0.83 & $\begin{array}{c}7.33 \\
5.67\end{array}$ & 5.10 & $\begin{array}{l}1.17 \\
167\end{array}$ & 2.83 & 0.83 & & 8.58 & 2.33 & 2.67 & 4.75 & 1.00 & 1.00 & & 2.27 & 4.58 & & 15.08 & 15.00 & 3.00 & $2.17(14)$ & $118(2)$ \\
\hline $\begin{array}{l}\text { 3.U. of Queensland } \\
4 . \mathrm{U} \text { of Surrey }\end{array}$ & $\begin{array}{l}70.88 \\
5718\end{array}$ & $\begin{array}{l}1.60 \\
0.33\end{array}$ & 5.67 & 8.08 & $\begin{array}{l}1.67 \\
2.83\end{array}$ & $\begin{array}{l}1.75 \\
462\end{array}$ & $\begin{array}{l}1.98 \\
0.50\end{array}$ & 0.25 & 6.90 & & 0.90 & 6.33 & 100 & 0.67 & 100 & 1.92 & $\begin{array}{l}7.67 \\
3.67\end{array}$ & $\begin{array}{l}1.60 \\
020\end{array}$ & 5.43 & 12.72 & 5.75 & $10.12(2)$ & $\begin{array}{ll}118(2) \\
1164)\end{array}$ \\
\hline $\begin{array}{l}\text { 4.U. of Surrey } \\
\text { 5.Bournemouth U. }\end{array}$ & $\begin{array}{l}57.18 \\
43.05\end{array}$ & 0.33 & $\begin{array}{l}1.92 \\
1.00\end{array}$ & $\begin{array}{l}7.37 \\
9.42\end{array}$ & $\begin{array}{l}2.83 \\
2.75\end{array}$ & $\begin{array}{l}4.62 \\
2.08\end{array}$ & $\begin{array}{l}0.50 \\
0.50\end{array}$ & & $\begin{array}{l}7.00 \\
2.00\end{array}$ & 0.67 & $\begin{array}{l}0.25 \\
2.17\end{array}$ & $\begin{array}{l}2.50 \\
4.33\end{array}$ & $\begin{array}{l}1.00 \\
1.25\end{array}$ & $\begin{array}{l}1.92 \\
1.55\end{array}$ & 1.00 & 0.58 & $\begin{array}{l}3.67 \\
4.67\end{array}$ & $\begin{array}{l}0.20 \\
0.50\end{array}$ & $\begin{array}{l}1.77 \\
3.20\end{array}$ & $\begin{array}{r}13.20 \\
6.38\end{array}$ & 7.12 & $\begin{array}{r}6.35(4) \\
1.23(26)\end{array}$ & $\begin{aligned} \begin{array}{r}1166(4) \\
74(5)\end{array} & \end{aligned}$ \\
\hline 6.Sun Yat-Sen U. & 41.25 & 0.67 & 3.00 & 8.00 & 2.67 & 1.67 & 1.17 & & 4.42 & & & 1.83 & 1.25 & 1.50 & 1.67 & 0.50 & 1.75 & & 0.33 & $\begin{array}{r}0.00 \\
12.08\end{array}$ & 0.50 & $\begin{array}{l}2.297(11) \\
2\end{array}$ & $74(5)$ \\
\hline 7.Texas A\&M U. & 39.83 & 1.50 & 0.25 & 2.75 & 1.20 & 2.83 & 1.00 & & 5.67 & 0.75 & 1.00 & 2.58 & 0.50 & & & 0.50 & 1.33 & 3.00 & 0.75 & 7.47 & 6.75 & $2.34(13)$ & $71(7)$ \\
\hline 8.U. of Florida & 37.48 & 1.67 & 9.35 & 8.03 & 1.00 & 0.83 & & 1.00 & 2.67 & 2.00 & 2.33 & 1.83 & & & & & 2.10 & 0.33 & & 4.33 & & $35(5)$ & $60(9)$ \\
\hline 9.Univ & 37.23 & & 1.25 & 3.92 & 1.50 & 1.25 & & 1.50 & 2.25 & 0.20 & 1.83 & & 0.50 & 1.00 & & & 3.28 & & 6.33 & 7.92 & 4.50 & (6) & $55(13)$ \\
\hline 10.Pur & 33.38 & 1.00 & 3.58 & 9.13 & & 2.33 & & 0.50 & 3.27 & 1.08 & & 0.70 & 0.50 & 0.25 & & 0.25 & 2.70 & & & 6.92 & 1.17 & $1.66(20)$ & $58(10)$ \\
\hline $\begin{array}{l}\text { 11.Temple University } \\
\text { 12 UCF }\end{array}$ & 33 & $\begin{array}{l}0.58 \\
0.20\end{array}$ & & 7.33 & & $\begin{array}{l}0.33 \\
150\end{array}$ & & 1.50 & $\begin{array}{l}3.83 \\
3.95\end{array}$ & 1.67 & 0.75 & 0.33 & 0.25 & & 0.50 & & 4.67 & 0.25 & & 9.25 & 2.08 & $1.14(27)$ & $67(8)$ \\
\hline $\begin{array}{l}\text { 12.UCF } \\
\text { 13.Virginia Tech }\end{array}$ & $\begin{array}{l}30.20 \\
29.80\end{array}$ & $\begin{array}{l}0.20 \\
0.33\end{array}$ & & $\begin{array}{l}6.12 \\
1.95\end{array}$ & $\begin{array}{l}1.20 \\
1.67\end{array}$ & 1.50 & & 0.67 & $\begin{array}{l}3.95 \\
3.85\end{array}$ & $\begin{array}{l}1.25 \\
1.17\end{array}$ & $\begin{array}{l}1.75 \\
1.42\end{array}$ & $\begin{array}{l}2.50 \\
1.20\end{array}$ & & 0.25 & & 1.25 & $\begin{array}{l}1.37 \\
2.25\end{array}$ & 0.67 & $\begin{array}{l}0.58 \\
3.0\end{array}$ & $\begin{array}{l}4.45 \\
8.97\end{array}$ & $\begin{array}{l}3.42 \\
3.08\end{array}$ & $\begin{array}{l}0.52(40) \\
1.98(15)\end{array}$ & $\begin{array}{l}57(12) \\
58(10)\end{array}$ \\
\hline 14.U. of Waikato & 28.28 & 1.00 & & 3.67 & 1.53 & & 1.81 & 0.01 & $\begin{array}{l}.808 \\
2.08\end{array}$ & & & $\begin{array}{l}1.20 \\
0.50\end{array}$ & 0.67 & $\begin{array}{l}0.25 \\
1.00\end{array}$ & & & 5.93 & 1.00 & & $\begin{array}{l}8.91 \\
4.42\end{array}$ & $\begin{array}{l}3.08 \\
4.67\end{array}$ & $\begin{array}{l}1.98(13) \\
14.14(1)\end{array}$ & $47(15)$ \\
\hline 15.Pennsylvania State & 27.00 & 0.50 & 1.50 & 4.33 & 4.58 & & & & 5.17 & & 0.83 & 0.67 & & & 0.25 & & 2.83 & & & 5.50 & 0.83 & $1.80(17)$ & $44(17)$ \\
\hline 16.Arizona State & 25.83 & & & 3.50 & 1.17 & & 1.00 & & 0.67 & & & 0.25 & & 1.00 & & & 8.92 & & 4.17 & 3.50 & 1.67 & $\mathrm{n} / \mathrm{a}$ & $42(18)$ \\
\hline puth Carolina & & 0.33 & 0.50 & & 1.08 & 0.53 & & & 2.77 & 2.95 & 0.33 & 1.60 & & & & & 0.90 & & 0.67 & & 2.17 & $0.65(37)$ & $48(14)$ \\
\hline 18.ULP & 21.83 & 0.33 & 1.00 & 5.50 & 1.33 & 2.50 & & 1.00 & 1.00 & 1.00 & 0.33 & & 1.50 & & & & 2.00 & & & 3.33 & 1.00 & & $29(25)$ \\
\hline $\begin{array}{l}\text { gton State } \\
\text { University }\end{array}$ & $\begin{array}{l}21.73 \\
21.33\end{array}$ & & 1.50 & $\begin{array}{r}3.00 \\
3.58\end{array}$ & 0.83 & & & & 1.50 & & 0.50 & 1.37 & 1.00 & 1.00 & & & 0.67 & & 0.58 & 7.78 & 2.00 & $1.35(22)$ & $46(16)$ \\
\hline $\begin{array}{l}\text { 20.Monanh University } \\
\text { 21.Kyung Hee }\end{array}$ & $\begin{array}{l}21.33 \\
21.32\end{array}$ & 1.00 & & $\begin{array}{l}3.58 \\
0.33\end{array}$ & 1.67 & 1.08 & 1.00 & 0.83 & $\begin{array}{l}1.33 \\
2.75\end{array}$ & $\begin{array}{l}1.00 \\
2.67\end{array}$ & 1.25 & $\begin{array}{l}1.00 \\
1.83\end{array}$ & 0.50 & 1.17 & & 0.33 & $\begin{array}{l}2.58 \\
3.42\end{array}$ & 0.75 & 1.50 & $\begin{array}{l}3.50 \\
7.32\end{array}$ & 0.25 & $\begin{array}{l}0.62(38) \\
0.76(36)\end{array}$ & $\begin{array}{l}38(19) \\
37(20) \\
-10\end{array}$ \\
\hline 22.B-G & 18.25 & 1.00 & & 2.42 & 0.25 & 1.00 & 0.50 & 0.50 & 1.67 & 1.00 & 0.50 & & & 1.00 & & & 2.33 & 1.00 & 0.50 & 4.58 & 3.00 & $9.12(3)$ & $25(32)$ \\
\hline n Cross & 17.20 & & 0.75 & 1.17 & 0.17 & 1.25 & 1.10 & 1.27 & 0.33 & & 1.50 & 1.00 & & & & & 1.33 & & 0.50 & 4.00 & 2.83 & $1.92(16)$ & $28(26)$ \\
\hline 24.Sejc & 17.15 & & & 1.08 & 0.50 & 0.50 & & & 2.67 & 0.50 & & 1.50 & & & & & 2.15 & & 0.25 & 7.75 & 0.25 & $0.55(39)$ & $33(21)$ \\
\hline ante & & & & 1.83 & & 3.83 & & 1.00 & 2.75 & 1.00 & & & 1.00 & 0.75 & & 1.00 & & & 00 & & & $\mathrm{n} / \mathrm{a}$ & $22(37)$ \\
\hline 26.V & & & & 1.50 & 2.50 & 2.33 & 0.14 & 0.33 & 0. & & 1.50 & 0.33 & & 0.33 & 0.50 & & 1.28 & 0.50 & 58 & & 0.33 & $\mathrm{n} / \mathrm{a}$ & $32(22)$ \\
\hline $\begin{array}{l}\text { 27.U. of Water } \\
28 \text { National Ch }\end{array}$ & $\begin{array}{l}15.83 \\
15.75\end{array}$ & 0.33 & 0.33 & $\begin{array}{l}2.33 \\
2.25\end{array}$ & & & 0.50 & & $\begin{array}{l}2.50 \\
0.67\end{array}$ & & & $\begin{array}{l}0.25 \\
025\end{array}$ & & 1.33 & & & 0.75 & 150 & 1.92 & $\begin{array}{l}3.08 \\
3.50\end{array}$ & 3.00 & $0.98(31)$ & $31(23)$ \\
\hline $\begin{array}{l}\text { 28.National Cheng Kung } \\
\text { 29.U. of Strathclyde }\end{array}$ & $\begin{array}{l}15.75 \\
15.62\end{array}$ & 0.33 & & $\begin{array}{l}2.25 \\
0.33\end{array}$ & 0.92 & $\begin{array}{l}0.33 \\
1.00\end{array}$ & $\begin{array}{l}0.50 \\
2.00\end{array}$ & & $\begin{array}{l}0.67 \\
0.33\end{array}$ & & & 0.25 & 1.00 & 0.33 & & & $\begin{array}{l}2.00 \\
4.92\end{array}$ & $\begin{array}{l}1.50 \\
1.25\end{array}$ & 4.75 & $\begin{array}{l}3.50 \\
3.03\end{array}$ & 0.17 & $\begin{array}{l}1.75(18) \\
1.73(19)\end{array}$ & $\begin{array}{l}22(37) \\
27(30)\end{array}$ \\
\hline h Wales & 15 & & & 2.62 & & 2.75 & & 1.78 & 1.33 & & 1.00 & 0.83 & 1.00 & 2.33 & & 0.50 & 0.25 & & & $\begin{array}{l}3.03 \\
1.00\end{array}$ & $\begin{array}{l}0.00 \\
1.00\end{array}$ & & $28(26)$ \\
\hline $31 . \mathrm{C}$ & & 0.53 & & 4.75 & 0.67 & 0.33 & 0.14 & & 1.00 & 0.50 & & 1.00 & & & & & & & 0.50 & 4.67 & 1.00 & & $30(24)$ \\
\hline 32.J. & 14 & 1.50 & 1.83 & & & 1.00 & 0.75 & & 1. & & & 3 & & & & & 1.00 & 0.50 & & 5.20 & 1.00 & & 25(33) \\
\hline & & & & 1.17 & & & & & & 1.50 & 1.00 & 1.70 & 1.00 & 0.25 & & & & & 3 & & & & $24(32)$ \\
\hline versity & 14 & & & 1.00 & 1.00 & & & & & & & & & & & & 2.00 & 0.75 & 1.67 & & .58 & $3.66(8)$ & $22(37)$ \\
\hline 35. & 14. & & & 0.58 & 0.33 & 0.25 & & 0.25 & 0.83 & 1.33 & 0.67 & & & 1.00 & & & 3.92 & & 0.67 & 3.00 & 50 & & $26(31)$ \\
\hline 36.7 & 13.43 & 0.50 & 1.33 & 0.25 & 0.67 & 1.00 & & & 1.75 & 0.33 & 0.33 & 0.33 & 0.33 & & & 0.50 & 0.20 & 033 & 0.20 & $\begin{array}{r}5.53 \\
400\end{array}$ & $\begin{array}{l}0.50 \\
167\end{array}$ & $1.03(28)$ & $\begin{array}{l}28(26) \\
25(32)\end{array}$ \\
\hline $\begin{array}{l}37.4 \\
38.4\end{array}$ & 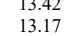 & & 2.25 & $\begin{array}{l}.1 .83 \\
1.33\end{array}$ & 0.33 & $\begin{array}{l}2.00 \\
1.00\end{array}$ & 0.92 & & $\begin{array}{l}2.0 \\
1.3 \\
\end{array}$ & $\begin{array}{l}0.33 \\
0.67\end{array}$ & $\begin{array}{l}0.25 \\
0.17\end{array}$ & & & & & & & $\begin{array}{l}0.33 \\
0.17\end{array}$ & 2.67 & & $\begin{array}{l}1.67 \\
1.00\end{array}$ & $\begin{array}{r}1.03(28) \\
3.29(9)\end{array}$ & $\begin{array}{l}25(32) \\
23(36)\end{array}$ \\
\hline & & & & 1.83 & 1.50 & & & 1.00 & 1. & & 0.25 & 0.33 & 0.33 & 1.00 & & & 2.33 & & 0.50 & 1.67 & 0.50 & & $22(37)$ \\
\hline & & 0.50 & 0.17 & 0.25 & 1.17 & 1.50 & & & 1. & & & & 1.50 & 1.00 & & & 0.25 & 1.00 & 1.25 & 3.33 & & $1.29(25)$ & $22(37)$ \\
\hline & & & 0.40 & & 0.25 & 2.00 & & & 2. & & 2.00 & & & & & & 1.00 & 1.00 & & & 2.00 & $n / a$ & 46) \\
\hline & 12. & & 1.67 & 4.00 & & & & & 1.00 & & & & 1.00 & & & & & & 1.67 & 1.00 & 17 & & $19(44)$ \\
\hline Technology & 12.50 & & 0.33 & & & 1.00 & 1.00 & & 1.83 & 0.33 & 0.25 & 1.00 & & & & & 367 & 3.00 & 0.92 & 3 & 50 & $1.56(21)$ & $15(46)$ \\
\hline $\begin{array}{l}\text { of Technology } \\
\text { achusets }\end{array}$ & $\begin{array}{l}12.42 \\
12.20\end{array}$ & 0.33 & 1.00 & 133 & & 0.75 & & 167 & & $\begin{array}{l}1.00 \\
0.50\end{array}$ & 05 & & 0.50 & & & & 2.67 & & & 0 & $\begin{array}{l}.00 \\
50\end{array}$ & $\begin{array}{l}1.03(28) \\
081(32)\end{array}$ & $\begin{array}{l}14(49) \\
2806)\end{array}$ \\
\hline achusetts & $\begin{array}{l}12.2 \\
12 .\end{array}$ & 0.33 & & $\begin{array}{l}.333 \\
0.58\end{array}$ & & 1.00 & & 1.67 & 2.8. & 0.50 & $\begin{array}{l}0.50 \\
2.00\end{array}$ & & 0.50 & & & & & & & $\begin{array}{l}3.53 \\
3.25\end{array}$ & .17 & $\begin{array}{l}0.81(32) \\
1.33(23)\end{array}$ & $\begin{array}{l}28(26) \\
15(46)\end{array}$ \\
\hline & 11 & 0.75 & & 1.42 & & & & & 1 & & 1.00 & & & & & & 2.00 & & 0.71 & 3.85 & 1.25 & & 5) \\
\hline eston & 11 & & 0.25 & 3.75 & & & & & 1.17 & 0.60 & 0.25 & & 1.25 & & 1.00 & & & & & 1.75 & 0.33 & & 43) \\
\hline 49.U. of Bologna & 11.83 & & & 5.33 & & $\begin{array}{l}1.50 \\
250\end{array}$ & & & 1.00 & & 100 & 1.00 & 2.00 & & & & $\begin{array}{l}1.00 \\
150\end{array}$ & 0.33 & & 24 & 117 & $0.78(34)$ & $14(49)$ \\
\hline
\end{tabular}

Econometrics; EDU = Education; GEO = Geographical Issue; GMKT = General Marketing; IMG = Image and Branding; IT = Information Technology; MICE = Meetings, Incentives, Conventions, and Exhibitions Including Festival and Fair; PLN = Tourism Planning; PPL = Politics, Policy, Legal, and Governmental Issue; SCM = Supply Chain Management; SEG = Segmentation; SIT = Special

Interests Tourism Such as Heritage, Farm, Cultural, Wine, or Food Tourism; SMT = Service Management; SUT = Sustainable Tourism and Ecotourism; TPB = Tourists' Perception and Behavior; OTH

= Others; U. = University; UCF = University of Central Florida; B-GUN = Ben-Gurion University of the Negev; UNLV = University of Nevada, Las Vegas; ULPGC = University of Las Palmas de Grad

Canaria. In the column of number of publications, the first number is the count of papers, and the second number in parentheses is the ranking based on the count of papers. 
Table 7. Country/Region Ranking and Research Areas of Tourism During 2010-2019

\begin{tabular}{|c|c|c|c|c|c|c|c|c|c|c|c|c|c|c|c|c|c|c|c|c|c|c|}
\hline $\begin{array}{l}\text { Country/Region Ranking } \\
\text { by Score }\end{array}$ & Score & ATT & CSM & DMKT & DVP & ECO & EDU & GEO & GMKT & IMG & IT & MICE & PLN & PPL & SCM & SEG & SIT & SMT & SUT & TPB & OTH & $\begin{array}{l}\text { Number of } \\
\text { Publication }\end{array}$ \\
\hline 1.USA & 657.08 & 10.20 & 25.17 & 98.38 & 21.70 & 33.28 & 5.25 & 9.17 & 76.02 & 20.72 & 17.50 & 24.83 & 11.42 & 10.00 & 3.25 & 4.00 & 71.86 & 9.58 & 34.96 & 127.54 & 42.25 & $876(1)$ \\
\hline 2.UK & 414.75 & 8.40 & 10.67 & 40.65 & 21.23 & 23.35 & 9.89 & 3.25 & 38.58 & 6.42 & 9.37 & 29.95 & 13.33 & 11.55 & 1.00 & 1.58 & 53.67 & 7.45 & 22.53 & 68.65 & 33.23 & $581(2)$ \\
\hline 3.Australia & 411.08 & 5.00 & 25.75 & 46.70 & 12.92 & 19.25 & 6.08 & 6.33 & 41.95 & 6.08 & 10.32 & 17.75 & 3.75 & 8.40 & 1.00 & 6.85 & 44.75 & 11.55 & 34.12 & 79.20 & 23.33 & $552(3)$ \\
\hline 4.Spain & 224.23 & 0.33 & 6.40 & 39.02 & 12.17 & 19.38 & 0.67 & 5.00 & 27.17 & 11.17 & 9.83 & 6.17 & 8.00 & 4.83 & 0.75 & 1.00 & 12.42 & 3.00 & 16.00 & 32.17 & 8.77 & $260(6)$ \\
\hline 5.Mainland China & 205.75 & 4.25 & 9.17 & 22.57 & 14.46 & 12.67 & 2.17 & 0.50 & 18.32 & 2.43 & 9.77 & 7.08 & 2.75 & 5.08 & 2.92 & 0.50 & 16.75 & 4.00 & 11.22 & 51.07 & 8.08 & $325(4)$ \\
\hline 6.Hong & 162.22 & 1.17 & 3.75 & 19.08 & 5.83 & 9.20 & 2.00 & 0.67 & 19.88 & 6.67 & 6.28 & 8.08 & 4.42 & 4.75 & 0.83 & 1.00 & 8.83 & 2.75 & 1.32 & 38.28 & 17.42 & $288(5)$ \\
\hline 7.Taiw: & 144.38 & 1.00 & 6.58 & 12.25 & 6.50 & 10.28 & 5.25 & & 13.08 & 1.78 & 3.25 & .03 & 1.50 & 3.00 & 2.00 & 2.00 & 13.95 & 10.00 & 16.17 & 29.00 & 2.75 & $178(7)$ \\
\hline 8.New Zealand & 111.04 & 1.20 & 2.75 & 14.78 & 3.78 & 3.33 & 4.02 & 2.00 & 8.52 & 0.20 & 2.83 & 1.00 & 5.42 & 3.17 & 0.50 & 1.33 & 11.85 & 1.50 & 6.67 & 22.10 & 14.08 & $167(8)$ \\
\hline 9.Korea & 88.88 & 1.50 & & 4.57 & 1.00 & 4.83 & 1.00 & & 12.42 & 4.33 & 2.25 & 5.92 & 2.25 & & & & 11.77 & 3.92 & 1.63 & 29.92 & 1.58 & $131(9)$ \\
\hline 10.Italy & 83.05 & 1.00 & 0.50 & 18.95 & 2.00 & 7.42 & & 4.00 & 10.17 & 1.00 & 2.92 & 5.83 & 5.00 & 1.75 & & 1.92 & 7.71 & & 3.13 & 8.75 & 1.00 & 101(11) \\
\hline 11.Ca & 67.69 & 0.33 & 0.58 & 6.17 & 3.92 & 1.83 & 2.00 & 1.33 & 7.42 & 0.20 & 0.25 & 2.48 & 0.50 & 1.98 & & & 6.97 & 2.42 & 7.24 & 16.07 & 6.00 & $110(10)$ \\
\hline 12.Netherlands & 58.86 & 0.50 & 2.25 & 6.60 & 2.83 & 3.17 & 0.83 & & 2.00 & 1.33 & 1.00 & & 2.67 & 3.05 & & 0.33 & 9.26 & 1.00 & 3.03 & 14.17 & 4.83 & $79(12)$ \\
\hline 13.Isra & 50.17 & 1.33 & 2.00 & 4.67 & 0.75 & 2.00 & 1.50 & 0.50 & 2.17 & 1.00 & 1.00 & & & 2.00 & & 0.50 & 10.17 & 1.00 & 3.00 & 14.25 & 2.33 & $62(15)$ \\
\hline 14.Austric & 44.77 & & 4.42 & 7.92 & & 3.65 & & 0.67 & 4.33 & 1.80 & 2.00 & 0.20 & 1.50 & & & 0.83 & 3.33 & 1.00 & 0.33 & 8.45 & 4.33 & $66(14)$ \\
\hline 15.Nor & 43.42 & 0.33 & 2.00 & 5.00 & 3.00 & & 2.00 & & 4.70 & & 0.60 & & & & & 2.33 & 3.83 & & 2.60 & 14.52 & 2.50 & $54(16)$ \\
\hline $16 . \mathrm{M}$ & 43.20 & 0.83 & 0.50 & 6.2 & 1.67 & 4.50 & 1.00 & & 2.87 & 0.67 & 1.75 & 3.83 & 1.50 & & 0.25 & & 4.75 & 1.83 & & 9.33 & 1.67 & 71(13) \\
\hline 17.M & 41.06 & 1.67 & 1.75 & 5. & 6.8 & 2.00 & 1.00 & & 1.33 & 0.33 & & & & & 1.00 & 0.65 & 7.13 & 1.00 & 1.00 & 9.73 & 0.67 & $51(18)$ \\
\hline 18.Portugal & 36.03 & & 2.00 & 3.00 & 0.75 & 4.37 & & & 5.08 & & & 2.00 & 0.50 & 2.50 & & & 4.33 & & 2.42 & 8.58 & 0.50 & $52(17)$ \\
\hline 19.Franc & 31.43 & & 1.33 & 6.17 & 0.25 & 2.57 & & 2.00 & 4.00 & & & 1.00 & & & & & 7.00 & 0.33 & 2.67 & 4.12 & & $47(19)$ \\
\hline 20. Turkey & 30.02 & 1.00 & & 2.87 & 2.17 & 3.58 & 0.33 & & 4.25 & 1.00 & 1.25 & 0.65 & 1.00 & 1.25 & 1.00 & 1.00 & 0.67 & & 3.00 & 4.00 & 1.00 & $45(20)$ \\
\hline
\end{tabular}

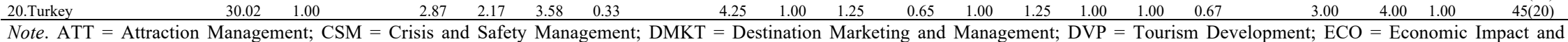
Note. ATT = Attraction Management; CSM = Crisis and Safety Management; DMKT = Destination Marketing and Management; DVP = Tourism Development; ECO = Economic Impact and
Econometrics; EDU = Education; GEO = Geographical Issue; GMKT = General Marketing; IMG = Image and Branding; IT = Information Technology; MICE = Meetings, Incentives, Conventions, and Exhibitions Including Festival and Fair; PLN = Tourism Planning; PPL = Politics, Policy, Legal, and Governmental Issue; SCM = Supply Chain Management; SEG = Segmentation; SIT = Special Interests Tourism Such as Heritage, Farm, Cultural, Wine, or Food Tourism; SMT = Service Management; SUT = Sustainable Tourism and Ecotourism; TPB = Tourists' Perception and Behavior; OTH $=$ Others 
Table 7 demonstrates the top 20 countries/regions in tourism during 2010-2019. The top three countries/regions got 1482.92 points at $44.2 \%$ of the total points of the tourism papers. Specifically, they were the United States (657.08 points, 19.6\%), the United Kingdom (414.75 points, 12.4\%), and Australia (411.08 points, 12.2\%). These top three countries/regions were consistent during both the first and second decades of the millennium.

\section{Research Rankings in Hospitality and Tourism Combined}

The hospitality and tourism papers were further combined for analysis. The total scores of the top 100 authors were 834.47 at $15.7 \%$ of the total points during 2010-2019, compared to 647.11 points at $22.8 \%$ of the total points during 2000-2009. The top three authors were SooCheong (Shawn) Jang, Rob Law, and Anna S. Mattila during both the first and second decades of the millennium. SooCheong (Shawn) Jang and Anna S. Mattila dominantly focused on hospitality research, while Rob Law contributed to hospitality and tourism equally.

The 100 most productive universities in the six journals gained 2775.74 points at $52 \%$ of the total points of all the research during $2010-2019$, in contrast to 1770.65 points at $62.5 \%$ of the total points of all the research during 2000-2009. The top three universities by the score during 20102019 were Hong Kong Polytechnic University (256.42 points, 4.4\%), Griffith University (104.93 points, $2 \%$ ), and Purdue University (103.42 points, 1.9\%). In contrast, during 2000-2009, the three most productive universities by the score were Cornell University, Hong Kong Polytechnic University, and Pennsylvania State University. By the number of publications, Hong Kong Polytechnic University ranked No.1 during both the first and second decades of the new millennium. And by weighted score, the most research efficient universities during 2010-2019 and 2000-2009 were the University of Waikato and College of Charleston, respectively.

The top three most productive countries/regions in hospitality and tourism research gained 2509.90 points at $46.8 \%$ of the total points. They were the United States (1510.57 points, $28.2 \%)$, the United Kingdom (502.10 points, 9.4\%), and Australia (497.23 points, 9.3\%). In contrast, during 20002009, the top three countries/regions were the United States, Hong Kong, and the United Kingdom.

\section{Conclusions and Implications}

Quality and quantity of publication output are undoubtedly an important benchmark of individual scholars, academic institutions, and intellectual advancement of countries/regions in a field. The periodical assessment of contributions to academic journals does reflect the changes of the hot research topics and shows who are contributing most to the advancement of a discipline through research and publications. Compared with the first decade of the new millennium, the six top-tier journals significantly augmented the capacity of publishing articles by increasing volumes and issues. The scholars who contributed to these journals were more diversified, which could be observed from the lower percentage of the scores that the top scholars gained. In the hospitality area, the top three scholars during 2010-2019 were still those who were reputed in productivity during 2000-2009. However, in the tourism area, two of the top three scholars during the second decade of the new millennium were rising stars after 2010.

From the perspective of research topics in hospitality, consistent with the results during 2000-2009, the studies on education and legal issues were still extremely deficient during 2010-2019. 
Additionally, green businesses relevant to environmental issues (e.g., legal issues, energy efficiency) have become popular in the middle to the late 2000s. However, those topics did not get the interest of scholars in the hospitality and tourism field. With the further development of green businesses during 2010-2019, more and more papers regarding environmentally-friendly practices (e.g., green hotels, green restaurants, sustainability tourism, and environmentally friendly tourism) have been published in hospitality and tourism journals. It is anticipated that increasing studies will emerge on the topic of green businesses in the hospitality and tourism field, considering the importance of global environmental protection issues. Moreover, food service got rare attention from scholars in the middle to the late $2000 \mathrm{~s}$, while the trend reversed in the $2010 \mathrm{~s}$. Due to the development of various foodservice industries (e.g., food tourism, food festival, food delivery, and cafeterias) and the high demand for the different restaurant industry types (e.g., fast-food, fastcasual, casual, and fine-dining restaurants), much research in foodservice paper has been published in six-top tier journals during 2010 to 2019.

The least popular themes in tourism during 2010-2019 were supply chain management and segmentation, while those themes during 2000-2009 were supply chain management and geographic issue. The number of studies relevant to the geographic issue significantly increased during the past ten years, probably because of the increasing applications of big data techniques in geography and the involvement of more scholars specialized in tourism geography. Similarly, tourism research in special interests such as heritage, farm, cultural, wine, and research in tourisms' economic impact and econometrics have still popular topics. For instance, wine, gastronomy, and a natural partnership in regional development have expanded into diverse aspects of tourism. Such topics of special interest play an essential part in tourism research because wine tourism and rural exploitation is relative heritage, farm, and culture for the long term.

Moreover, with the emergence of online review platforms since 2007, customers have a new information resource in the purchase decision-making process. From 2010 to 2019, the offerings of hospitality and tourism were shown in diverse third-party review websites (e.g., Airbnb, TripAdvisor, and Yelp). Consistently, the number of the papers in information technology and management systems published in the six top-tier hospitality and tourism journals increased from 47 during 2010-2009 to 154 during 2010-2019. Online reviews were an increasingly important venue for scholars to understand customers' perceptions and attitudes toward a business, brand, or product/service. Lastly, it needs to be noticed that five of the six top-tier journals (i.e., JHTR, IJHM, TM, ART, JTR increased volumes and issues per year. Especially IJHM has expanded publications almost two times (65 issues vs. 37 issues) compared 2010s with 2000s.

The number of studies related to segmentation dramatically decreased during the past ten years, probably due to the maturity of segmentation methods which leads to less interest of scholars. Overall, the present authors suggested that the six top-tier journals could consider initiating more special issues to motivate scholars to do more studies on education, legal issues, and supply chain management.

\section{Limitations and Future Research}

The present study has the following limitations. First, for the comparison purpose, we adopted the same research themes proposed by Park et al. (2011). However, during the past ten years, some new themes (e.g., sharing accommodation) emerged. We merged them into the most closing 
themes indicated in Park et al. (2011). These emerging topics may become independent themes when they become mature in the future. Therefore, reevaluation of the themes is necessary for future review studies. Second, we improved the procedure of identifying research themes used by Park et al. (2011) by involving two independent coders and checking inter-rater reliability. However, such a process may still be inherently biased. Future studies could consider content analysis as a supplement of identifying research themes. Third, the six journals could be revisited at the end of the next decade with the same method of Park et al. (2011) and the present study in order to continuously present a historical pattern of hospitality and tourism research. Fourth, the rankings of the journals via impact scores in the different indexes vary significantly. Even for the same index, the rankings change year by year. As a follow-up study of Park et al. (2011), we reviewed the same seven journals. However, in recent years, CHQ and JHTR as top-tier journals in the HTM field have been critiqued. Scholars should re-evaluate the positions of CHQ and JHTR in review studies.

\section{References}

Adler, N., Friedman, L., \& Sinuany-Stern, Z. (2002). Review of ranking methods in the data envelopment analysis context. European Journal of Operational Research, 140(2), 249-265.

Becker, C. (2017). Toward increased accuracy in productivity measurement: Evidence-based analytics. In M. Uysal, Z. Schwartz, \& E. Sirakaya-Turk (Eds.), Advanced in hospitality and tourism: Management Science in hospitality and tourism, theory, practice, and application (pp. 345-363). Apple Academic.

Çakmak, E., Lie, R., \& McCabe, S. (2018). Reframing informal tourism entrepreneurial practices: Capital and field relations structuring the informal tourism economy of Chiang Mai. Annals of Tourism Research, 72, 37-47.

Chan, E. S., \& Hsu, C. H. (2016). Environmental management research in hospitality. International Journal of Contemporary Hospitality Management, 28(5), 886-923.

Cunill, O., Salvá, A., Gonzale, L. O., \& Mulet-Forteza, C. (2019). Thirty-fifty anniversary of the international journal of hospitality management: A bibliometric overview. International Journal of Hospitality Management, 78, 89-101.

Gursoy, D., \& Sandstrom, J. (2016). An updated ranking of hospitality and tourism journals. Journal of Hospitality and Tourism Research, 40(1), 3-18.

Harris, P. J., \& Brown, J. B. (1998). Research and development in hospitality accounting and financial management. International Journal of Hospitality Management, 17(2), 161-182.

Ip, C., Law, R., \& Lee, H. A. (2011). A review of website evaluation studies in the tourism and hospitality fields from 1996 to 2009. The International Journal of Tourism Research, 13(3), 234-265.

Jogaratnam, G., Chon, K., McCleary, K., Mena, M., \& Yoo, J. (2005a). Analysis of institutional contributors to three major academic tourism journals: 1992-2001. Tourism Management, 26(5), 641-658.

Jogaratnam, G., Chon, K., McCleary, K., Mena, M., \& Yoo, J. (2005b). Journal of Hospitality and Tourism Research, 29(3), 356-371.

Jones, P., \& Lockwood, A. (1998). Operations management research in the hospitality industry. International Journal of Hospitality Management, 17(2), 183-202.

Kim, C. S., Bai, B. H., Kim, P. B., \& Chon, K. (2018). Review of reviews: A systematic analysis of review papers in the hospitality and tourism literature. International Journal of Hospitality Management, 70, 49-58.

Law, R., Leung, D., \& Cheung, C. (2012). A systematic review, analysis, and evaluation of research articles in the Cornell hospitality quarterly. Cornell Hospitality Quarterly, 53(4), 365-381.

Line, N. D., \& Runyan, R. C. (2012). Hospitality marketing research: Recent trends and future directions. International Journal of Hospitality Management, 31(2), 477-488.

Lu, J., \& Nepal, S. K. (2009). Sustainable tourism research: An analysis of papers published in the Journal of Sustainable Tourism. Journal of Sustainable Tourism, 17(1), 5-16.

Manoharan, A., \& Singal, M. (2017). A systematic literature review of research on diversity and diversity management in the hospitality literature. International Journal of Hospitality Management, 66, 77-91.

McKercher, B. (2012). Influence ratio: An alternate means to assess the relative influence of hospitality and tourism journals on research. International Journal of Hospitality Management, 31(3), 962-971. 
McKercher, B., Law, R., \& Lam, T. (2006). Rating tourism and hospitality journals. Tourism Management, 27(6), 1235-1252.

Min, H., Park, J., \& Kim, H. J. (2016). Common method bias in hospitality research: A critical review of literature and an empirical study. International Journal of Hospitality Management, 56, 126-135.

Nusair, K., Butt, I., \& Nikhashemi, S. R. (2019). A bibliometric analysis of social media in hospitality and tourism research. International Journal of Contemporary Hospitality Management, 31(7), 2691-2719.

Olsen, M. D., \& Roper, A. (1998). Research in strategic management in the hospitality industry. International Journal of Hospitality Management, 17(2), 111-124.

Park, K., Phillips, W. J., Canter, D. D., \& Abbott, J. (2011). Hospitality and tourism research rankings by author, university, and country using six major journals. Journal of Hospitality and Tourism Research, 35(3), 381416.

Ryan, C. (2005). The ranking and rating of academics and journals in tourism research. Tourism Management,26(5), 657-662.

Serra-Cantallops, A., Peña-Miranda, D. D., Ramón-Cardona, J., \& Martorell-Cunill, O. (2018). Progress in research on CSR and the hotel industry (2006-2015). Cornell Hospitality Quarterly, 59(1), 15-38.

Severt, D. E., Tesone, D. V., Bottorff, T. J., \& Carpenter, M. L. (2009). A world ranking of the top 100 hospitality and tourism programs. Journal of Hospitality \& Tourism Research, 33(4), 451-470.

Sheldon, P. J. (1991). An authorship analysis of tourism research. Annals of Tourism Research, 18(3), $473-484$.

Shen, Y., Morrison, A. M., Wu, B., Park, J., Li, C., \& Li, M. (2018). Where in the world? A geographic analysis of a decade of research in tourism, hospitality, and leisure journals. Journal of Hospitality \& Tourism Research, 42(2), 171-200.

Singh, N., Hu, C., \& Roehl, W. S. (2007). Text mining a decade of progress in hospitality human resource management research: Identifying emerging thematic development. International Journal of Hospitality Management, 26(1), 131-147.

Svensson, G., Svaeri, S., \& Einarsen, K. (2009). 'Empirical characteristics' of scholarly journals in hospitality and tourism research: An assessment. International Journal of Hospitality Management, 28(3), 479-483.

Tracey, J. B. (2014). A review of human resources management research: The past 10 years and implications for moving forward. International Journal of Contemporary Hospitality Management, 26(5), 679-705.

Zhou, P., \& Leydesdorff, L. (2011). Fractional counting of citations in research evaluation: A cross-and interdisciplinary assessment of the Tsinghua University in Beijing. Journal of Informetrics, 5(3), 360-368. 